\section{AN ANNOTATED COMPILATION OF CHILDHOOD PERVASIVE DISINTEGRATIVE SPECTRUM DISORDERS(PDD) CHECK LISTS WITH EMPHASIS ON DIFFERENTIAL ALGORITHMIC,DIAGNOSTIC THERAPEUTIC- INTERVENTIONAL APPROACH}

\section{E.U. Onyekwelu, Neuroscience}

\section{Paediatrics, Royal Victoria Teaching Hospital, Banjul, The Gambia}

Background-Historical-notes-purpose: PDDs are-gradable-constellations of neurolobiologicaldisorders with abbreviated-life -expectancies, including CDD, its anecdotal-extreme-rarity made it initially unscientifically-acknowledged-Rettssyndrome(RS)-affects only females.Previously most girls diagnosed asCDD, retrospectively had RS.In addition to Autism-spectrum-disorders(ASD),Aspergers syndrome(AS).An unequivocal initial diagnosis of a specific PDD was unusual, because of their striking overlapping features. Although features were spectral-overlapping,they were aetiologicallyprognostically distinct.Accurate-diagnosis is crucial because interventions-differ. The compositeroles of prematurities-birth- traumas-genetic factors-amyloid-interleukins-1-beta-endorphinsenvironmental- toxigenic- autoimmunityrelated disruption of neuronal-transmissionsneuro-pathological associations with TSC, -intrauterineTORCHES-viral-exanthematousrelated-SSPE-associated-symptomatic-seizures which suggests the-diagnosis of otherwise-similarbut- very -therapeutically-responsive-LandauKleffner-syndrome(LKS) especially in boys-the influence of other-infective-processes suggests the benefits of an apposite index of suspicion for a timely diagnosis/-specific-interventions.Ongoing research although undermined by the relative-rarity of these disorders-suggests that overall,PDDswere underdiagnosed-misdiagnosed.However the putative-roles of infective-antecedents suggests that impacts could be epidemio-geographically distinct.

A delineation of categorical-figures from a definedsetting-could be scientifically -illuminating with implications for research-directions.

Methods-case-definitions-interventions: Descriptors- pre-morbid-post morbid- historical notes were retrieved from teachers-care giversparents of pervasive-developmental-educationalsocial-emotional- physical aspects of cases-relevant to the theme.Putatively-universally-acceptablesymptomatic-syndrome-driven-classifiers with batteries of developmental-tests were applied for

Case-definitions.Interventions were structuredbehavioural-educational-speech-language therapies-social skills-development-sensoryintegration- occupational therapies-hypnoticsedatives-antiepileptics-tranqulizers. Rewards to reinforce desirable behaviours-discouraginguntoward-behaviours-Cares in respite-homes were proffered.

Results: Of the $(n=13)$ cases- $(n=8)$ were males. Mean-age at diagnosis $=89.08$ months.

On the basis of epidemiological-historicaldemographics-temporality-chronologyinterventional-responsiveness of symptomatologies, the diagnosis of compatibleearly infantile-autism-spectrum-disorders was suggested in $(n=1)$, compatible high functioning autism. $(n=1) \operatorname{CDD}(n=1)$, Retts-syndrome $(n=1)$. PPD-NOS ( $n=9)$-no compatible- AS.UnprovokedRemote-symptomatic-seizures were frequent $(n=11)$ Other associations were primary enuresis-socialdeviance-defective-self-regulation-derangedsleep-wake cycles-acute-psychosis-inordinatetemper-tantrums-non-specific-abdominal-painshallucinations-recurrent- febrile-responses-irritablebowel-syndromes-multiphasic-hallucinations-poor co-operation-attention span-concentrations/syncope.

Conclusions: In PPD, because outcomes were improved with earlier interventions, an opportunistic screening for developmental-defects should be undertaken in every well baby-child visits.Although symptomatically similar,- patterns of onset-courseoutcomes were categorically distinct.A consistentpositive approach results in improved outcomes. These figures could direct-diagnostic-therapeuticapproaches/-relevant interventions.

571

EFFECT OF NEONATAL HYPOXIA/ISCHEMIA

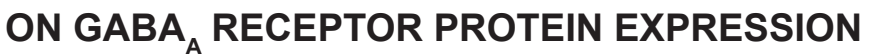

S.T. Bjorkman, Z. Ireland, P.B. Colditz, S.M. Miller

Perinatal Research Centre, UQ Centre for Clinical Research, The University of Queensland, Brisbane, QLD, Australia

Background and aims: Current therapeutic options for neonatal hypoxia/ischemia $(\mathrm{HI})$ are 